\title{
Characterization of Glass Delamination by TEM: Results from a New Sample Preparation Technique
}

\author{
Elaine F. Schumacher, Heidi M. Talesky, and Kristie J. Diebold
}

McCrone Associates, Inc., Westmont, IL 60559

analysis@mccrone.com

Abstract: A simple and efficient filtration method is presented for isolation of thin particulate from liquid pharmaceutical products directly onto grids for analysis by transmission electron microscopy with energy dispersive $x$-ray spectrometry (TEM/EDS). The method is applied to the problem of glass delamination, wherein a liquid drug product reacts with glass packaging, contaminating the product with glass flakes and residues. The filtration method facilitates preparation of representative samples for TEM/EDS, a technique shown to have advantages over scanning electron microscopy (SEM) with EDS for unambiguous characterization of thin particulate formed by glass delamination processes.

\section{Introduction}

Glass delamination is a serious and costly problem for pharmaceutical manufacturers and has led to several drug recalls in recent years. Delamination occurs when injectable solutions and suspensions react with glass packaging, resulting in contamination of drug products with glass flakes and residues formed during glass dissolution. Reaction of glass with a drug may also lead to formation of secondary products. The severity of glass delamination depends on several factors, including glass composition, presence of defects, glass container manufacturing methods, sterilization techniques, and drug storage conditions. Characteristics of the drug product such as $\mathrm{pH}$ or presence of buffers will also influence the glass delamination process.

A variety of analytical techniques may be used to verify that glass delamination has occurred, beginning with examination of the liquid drug product in the glass container, both visually and using a stereomicroscope. Particulate and fine residues are then typically isolated from liquid products by filtration and examined using light microscopy. The filtered particulate may then be analyzed using scanning electron microscopy with energy dispersive $\mathrm{X}$-ray spectrometry (SEM/EDS), a technique recommended for use in glass container screening studies as outlined in $\mathrm{USP}<1660>[1,2]$. The interior surfaces of empty glass containers may also be characterized by wrapping them with tape and breaking them to expose inner surfaces while maintaining the spatial relationship of the fragments. This is critical because specific areas such as the base or neck of a vial are more susceptible to delamination because of stresses incurred during the manufacturing process. Interior surfaces may exhibit possible delamination precursors such as etching and pitting. If the process is more advanced, flaking glass will be evident when surfaces are examined using light microscopy or SEM.

In some cases, visual examination, light microscopy, and SEM imaging may be sufficient to observe the presence of glass delamination. However, elemental analysis is key to differentiating possible delamination products, including glass flakes, coating fragments, dissolved glass residue, or secondary products formed by reaction between a drug component and a glass container. The most commonly used technique for obtaining compositional information about delamination materials is SEM/EDS, but there are some drawbacks to this approach. Removal of extremely thin samples such as delamination flakes or residues from filters for transfer to SEM substrates can be difficult. An alternative is to mount the entire filter on a substrate. Either preparation technique typically results in EDS spectra dominated by a signal from the substrate or the filter material, which is often polycarbonate (PC), and causes much smaller peaks for major elements present in the target thin sample (Figure 1). Peaks for minor or trace elements may be entirely absent, thereby lessening the value of SEM/EDS for discrimination between glass and other materials that may be present.

Transmission electron microscopy (TEM) with EDS has been shown to offer advantages over SEM/EDS for analysis of very thin particulate resulting from delamination of glass vials
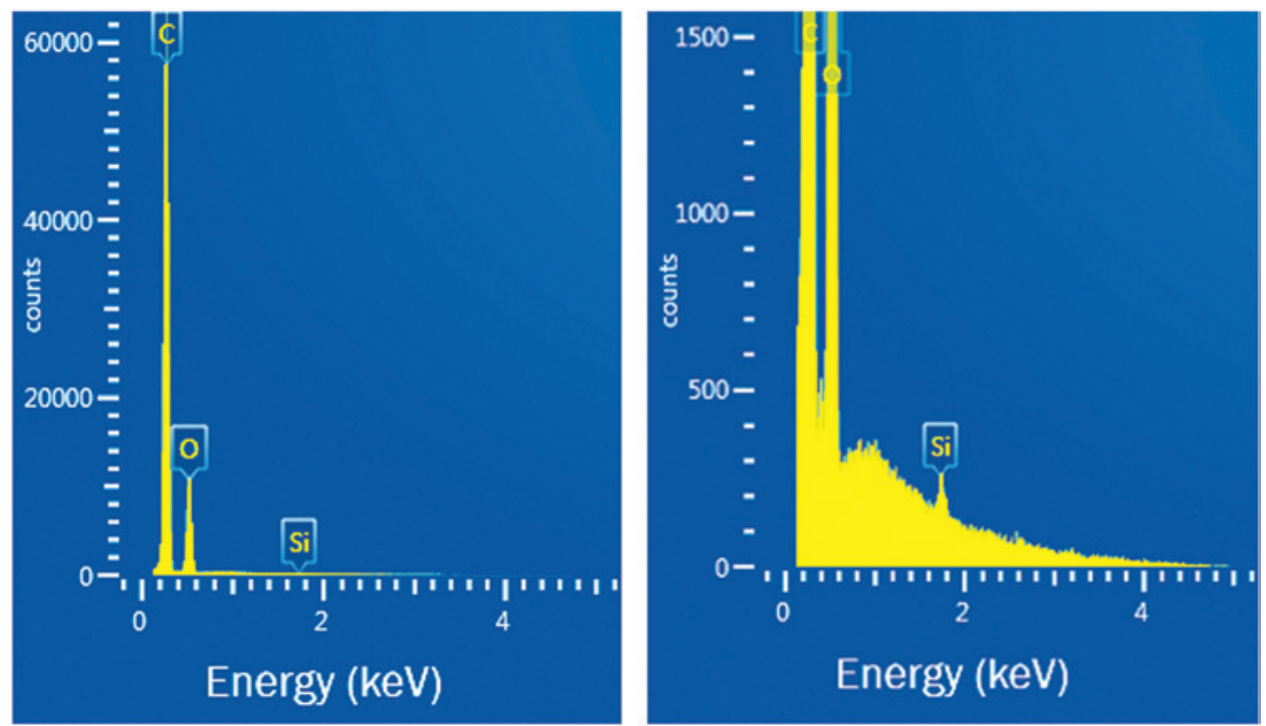

Figure 1: SEM/EDS spectrum acquired at $5 \mathrm{kV}$ from glass delamination flake on polycarbonate filter (left), and same spectrum with $y$-axis expanded to show peaks from glass components $\mathrm{Si}$ and $\mathrm{O}$ (right). 


\section{Imaging at}

sub-nanometer resolution.

\section{ZEISS GeminiSEM}

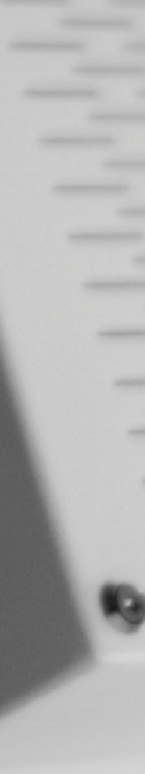

//INSPIRATION
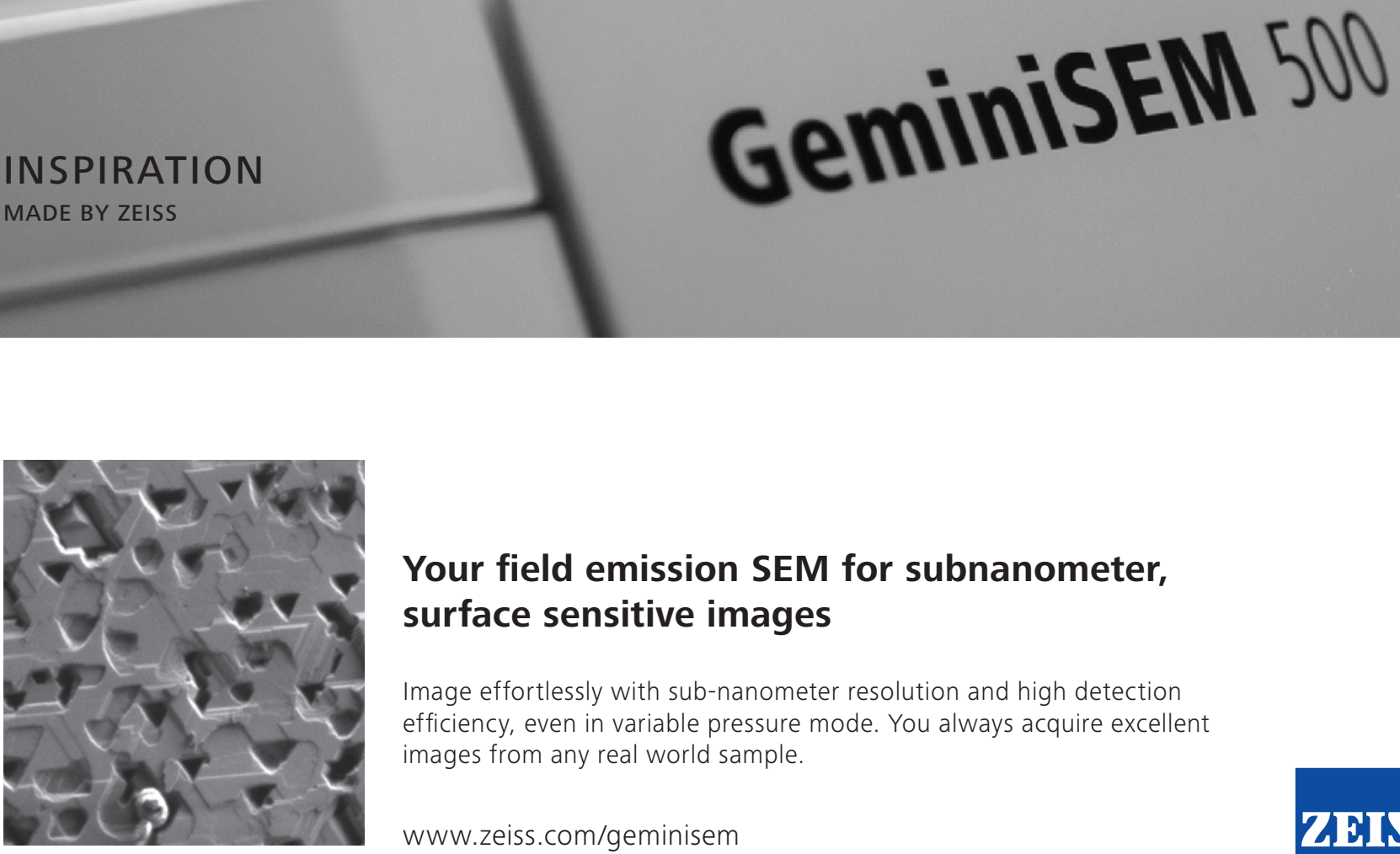

Your field emission SEM for subnanometer, surface sensitive images

Image effortlessly with sub-nanometer resolution and high detection efficiency, even in variable pressure mode. You always acquire excellent images from any real world sample. 


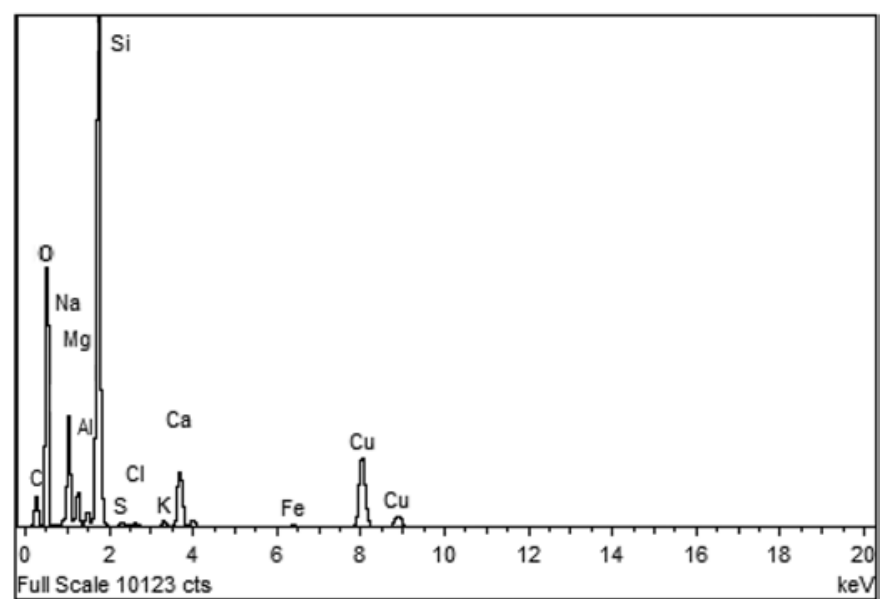

Figure 2: TEM/EDS spectrum from glass delamination flake on copper grid, clearly showing peaks for major, minor, and trace glass components.

and syringes [3]. Such samples are usually electron-transparent as isolated and require no further thinning, particularly when analysis is done in a $200-300 \mathrm{kV}$ materials science TEM. A representative TEM spectrum acquired from a glass delamination flake displays major silicon and oxygen peaks, as well
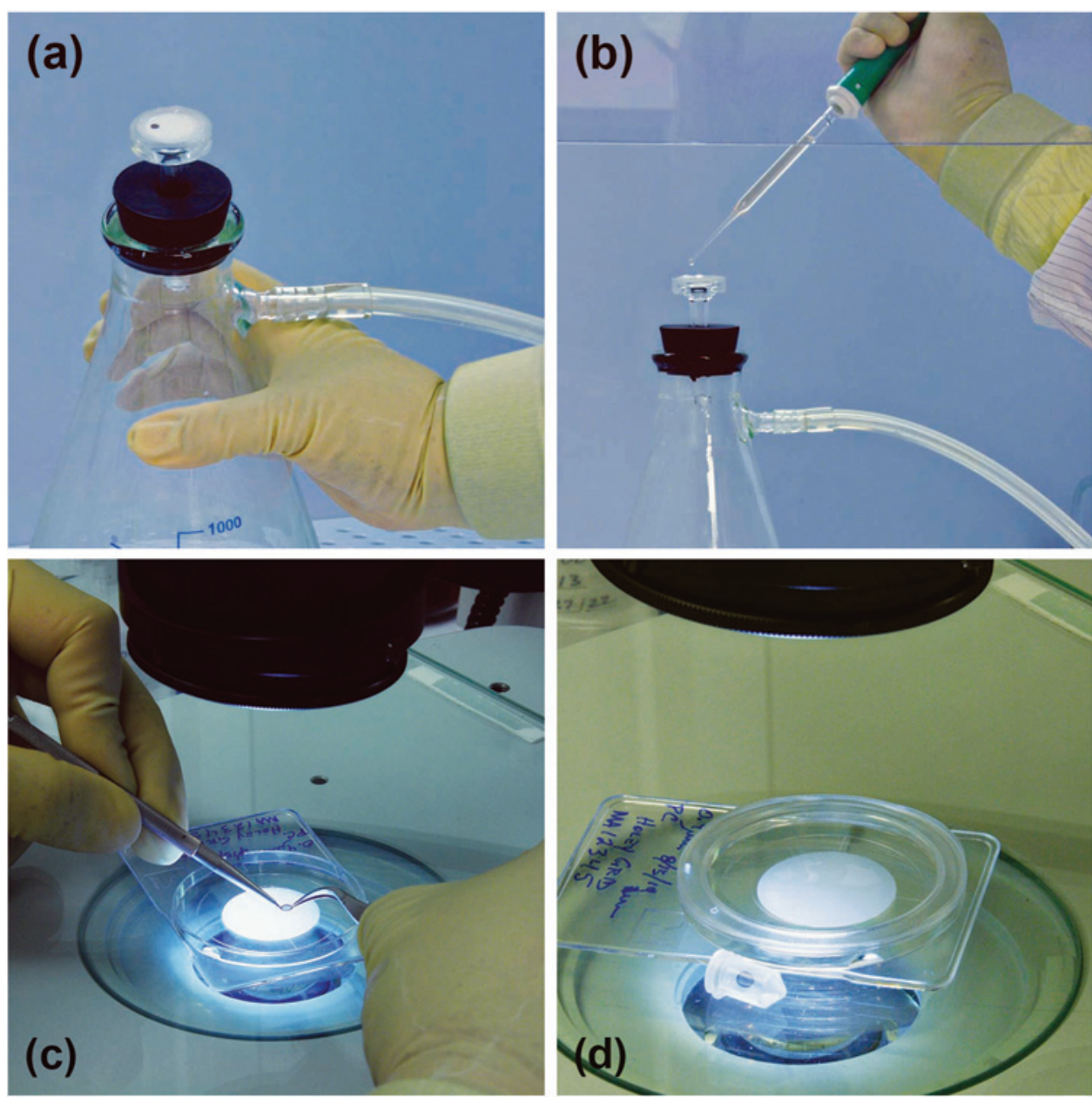

Figure 3: (a) Holey carbon-coated TEM grid positioned on polycarbonate filter. (b) Dropwise application of liquid to TEM grid on filter. (c) Polycarbonate filter transferred to Petri dish and viewed with light microscope for removal of grid. (d) TEM grid and filter for SEM examination prepared from the same filtration.
Table 1: Comparison of SEM and TEM results for characterization of particulate from liquid samples suspected to contain glass delamination flakes. SEM and TEM samples were simultaneously prepared on the same filter.

\begin{tabular}{|c|l|l|}
\hline Sample & \multicolumn{1}{|c|}{ Vial 1 SEM } & \multicolumn{1}{|c|}{ Vial 1 TEM } \\
\hline A & $\begin{array}{l}\text { No flakes } \\
\text { observed }\end{array}$ & $\begin{array}{l}\text { Two delamination } \\
\text { flakes }\end{array}$ \\
\hline B & $\begin{array}{l}\text { No flakes } \\
\text { observed }\end{array}$ & $\begin{array}{l}\text { Two delamination } \\
\text { flakes }\end{array}$ \\
\hline C & $\begin{array}{l}13 \text { glass flakes } \\
\text { observed }\end{array}$ & $\begin{array}{l}\text { One large } \\
\text { delamination flake }\end{array}$ \\
\hline D & $\begin{array}{l}\text { No flakes } \\
\text { observed }\end{array}$ & $\begin{array}{l}\text { A few C-rich } \\
\text { particles, two } \\
\text { possibly graphite }\end{array}$ \\
\hline
\end{tabular}

as peaks for minor and trace elements that can aid in identification of specific glass formulations (Figure 2).

Though the TEM is ideal for characterization of these very thin samples, transfer of flakes and residues from filters to TEM grids can be challenging. A method has been developed whereby filtration of liquid products directly onto holey carbon-coated TEM grids captures a sufficient amount of representative material to identify particulate isolated from liquid products.

\section{Materials and Methods}

Filtrations were carried out in a hood in an ISO Class 5 cleanroom, which provided a particle-free environment for sample preparation. To prepare blank grids and samples from liquids suspected to contain glass delamination flakes, a holey carbon-coated $3 \mathrm{~mm}$ TEM grid was positioned off-center on a PC filter placed on a fritted surface on a standard filtration apparatus (Figure 3a). Liquid was applied dropwise to the grid and to the center of the filter (Figure 3b). When filtration was complete, the PC filter was carefully lifted off of the fritted filter and placed in a disposable Petri dish. While observing under a light microscope, the grid was picked up from the filter using forceps and transferred to a storage capsule (Figure 3c). Using this procedure, samples suitable for both SEM and TEM analysis were prepared simultaneously (Figure 3d).

Blank samples were prepared using particle-free water to ensure that no particulate observed on TEM grids prepared by this method had come from the cleanroom environment or any equipment used for the filtration. As-received grids were also examined for background particulate. TEM grids 

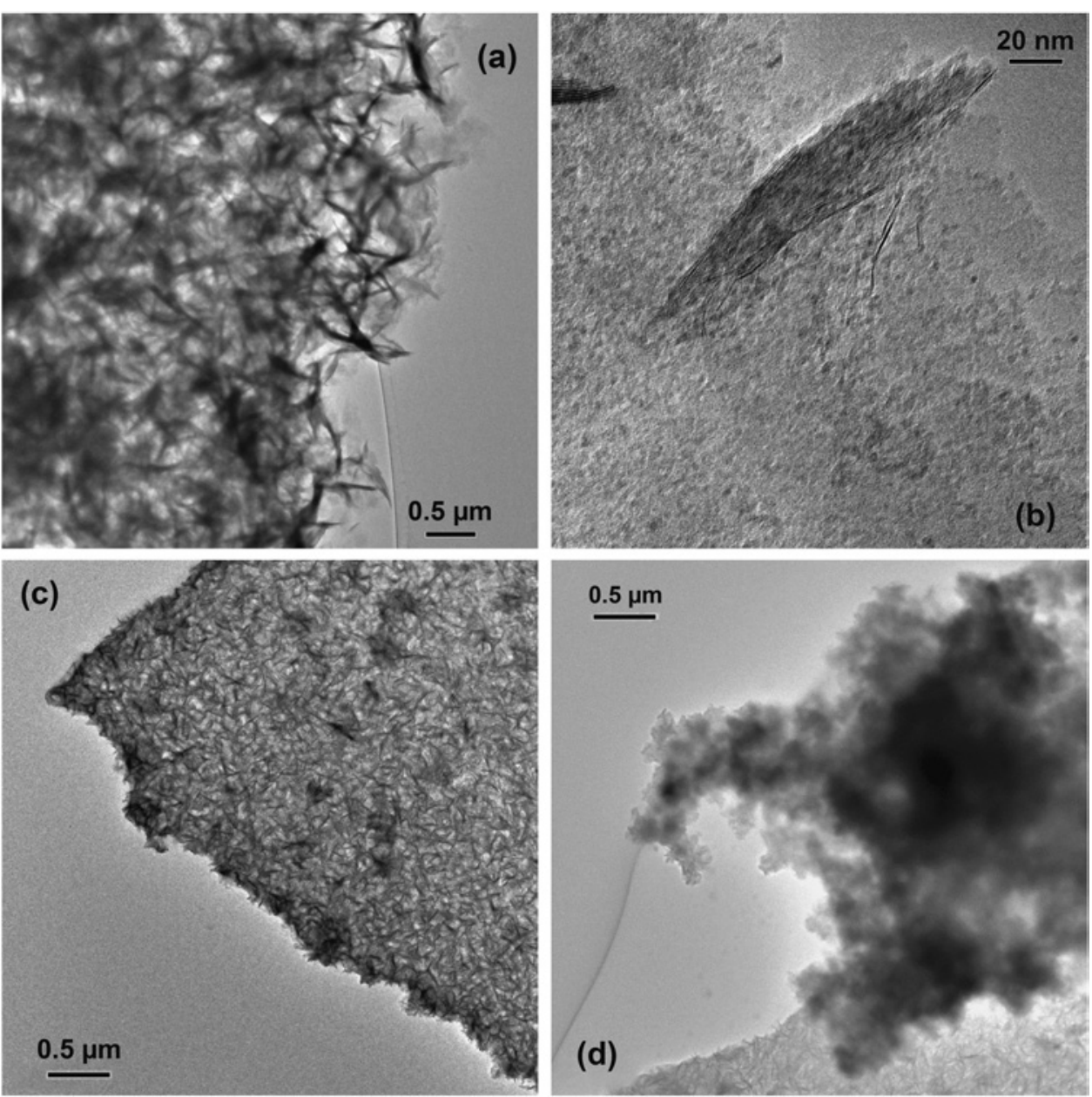

Figure 4: (a) Iron silicate particulate from iron sucrose drug product, filtered directly onto TEM grid. (b) High-magnification image of directly filtered iron silicate showing evidence of layered structure. (c) Layered iron silicate particulate from iron sucrose drug product, transferred to TEM grid using needle. (d) Amorphous iron silicate particulate from iron sucrose drug product, transferred to TEM grid using needle.
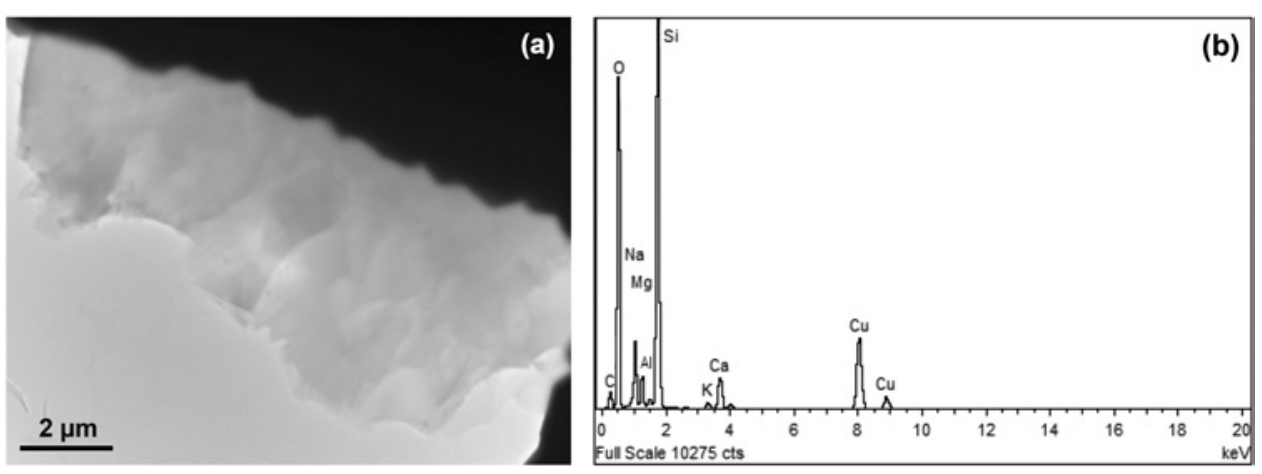

Figure 5: (a) Large glass delamination flake captured on grid bar by filtration directly onto TEM grid. (b) TEM/EDS spectrum from large delamination flake in (a) revealing glass components $\mathrm{Si}, \mathrm{O}, \mathrm{Ca}, \mathrm{Na}, \mathrm{K}, \mathrm{Al}$, and $\mathrm{Mg}$.

prepared by filtration of particlefree water were examined, and nothing was found that was consistent with materials commonly associated with glass delamination. For comparison to earlier work in which material was directly transferred from filters to TEM grids using fine needles, particulate was filtered directly onto a grid from an iron sucrose product used in a previous study. Characteristics of particulate isolated by filtration were consistent with those observed in material prepared by direct transfer. In addition to glass delamination flakes, two iron silicates with distinctly different morphologies and iron-to-silicon ratios were found, as was the case in the earlier work (Figures $4 \mathrm{a}-4 \mathrm{~d}$ ). One of these products had a layered structure and yielded measurable electron diffraction patterns, whereas the other was amorphous. These iron silicates are examples of materials that may result from dissolution of container glass and subsequent reaction with a drug component to form a secondary product.

SEM versus TEM analysis. A comparison of SEM and TEM results for four samples prepared by simultaneous filtration onto grids and PC filters is shown in Table 1. For Samples A and B, glass delamination flakes were captured on TEM grids but were not found on the associated PC filters examined in the SEM. Results for Samples C and D were consistent between the techniques, with a positive identification in one case (C) and a negative finding in the other (D). Overall, more complete information about particulate elemental composition was obtained by TEM/EDS analysis of material captured on grids.

TEM/EDS analysis. One delamination flake isolated from Sample A was quite large and had landed on a grid bar, which was often observed

coated with continuous carbon film were found to be unsuitable for the filtration procedure, as there was significantly more breakage of the film than was observed when grids with holey carbon coatings were used.

\section{Results}

Comparison to previous work. Little to no background particulate was found when as-received grids and blank grids to be the case for large flakes (Figure 5a). The large area of the thin flake that overhung the grid bar presented several areas suitable for acquisition of EDS spectra, despite being in close proximity to the grid (Figure $5 \mathrm{~b}$ ). In this case, the flake contained typical glass components $\mathrm{Si}, \mathrm{O}, \mathrm{Ca}, \mathrm{Na}, \mathrm{K}$, $\mathrm{Al}$, and $\mathrm{Mg}$.

In Sample B, a small cluster of thin fragments was found on the TEM grid film (Figure 6a), and EDS analysis confirmed 
a glass composition (Figure 6b). It is unlikely that a particle this small and thin would have had sufficient contrast to be found by SEM, even if it had it been isolated directly onto a polycarbonate filter.

Other types of small particles were found in glass delamination samples filtered onto TEM grids. In one sample, small heterogeneous particles containing elements typical of silicate and steel were found (Figures $7 \mathrm{a}-7 \mathrm{c}$ ), in addition to glass delamination flakes.

\section{Discussion}

Examination by SEM/EDS is an accepted technique for elemental analysis of glass delamination flakes and other thin particles and residues that form during glass dissolution. SEMs are readily available in many laboratories, and mounting of filters containing isolated particulate is straightforward. However, thin particles and residues may lack sufficient contrast to be easily located by SEM imaging. Because the beam/sample interaction volume in the SEM includes the thin sample and the substrate below it, SEM/EDS spectra obtained from glass delamination products are typically dominated by signals from the support materials, even when a low accelerating voltage is used. Peaks for major components of the particles, such as silicon, may be barely distinguishable above background, and peaks for minor and trace elements will typically not be detected.

Previous work has shown that TEM/EDS analysis is a more suitable approach, given that glass delamination particulate is thin enough to be electron transparent. Examinations of as-received grids and blank grids prepared by filtration of particlefree water indicate that background particulate is minimal and is unlikely to impact identification of materials associated with glass delamination processes. Good quality $\mathrm{x}$-ray spectra containing major, minor, and trace element peaks are easily acquired and may allow for better identification of glass product composition. Smaller particles and flakes that might be missed in an SEM survey of a filter are more likely to be found in the TEM, where high-resolution imaging, high-spatial-resolution EDS, and electron diffraction techniques can be brought to bear on the challenge of small particle identification. $\mathrm{Ca}, \mathrm{Al}$, and $\mathrm{K}$.
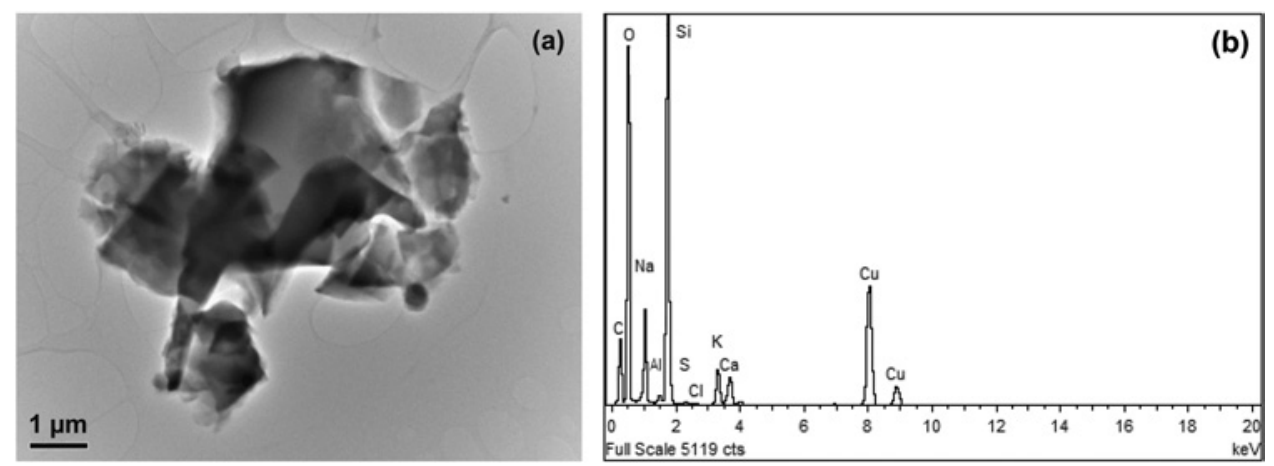

Figure 6: (a) Small cluster of glass delamination fragments captured on grid film by filtration directly onto TEM grid. (b) TEM/EDS spectrum from small glass delamination fragments in (a) revealing glass components $\mathrm{Si}, \mathrm{O}$, $\mathrm{Na}$,
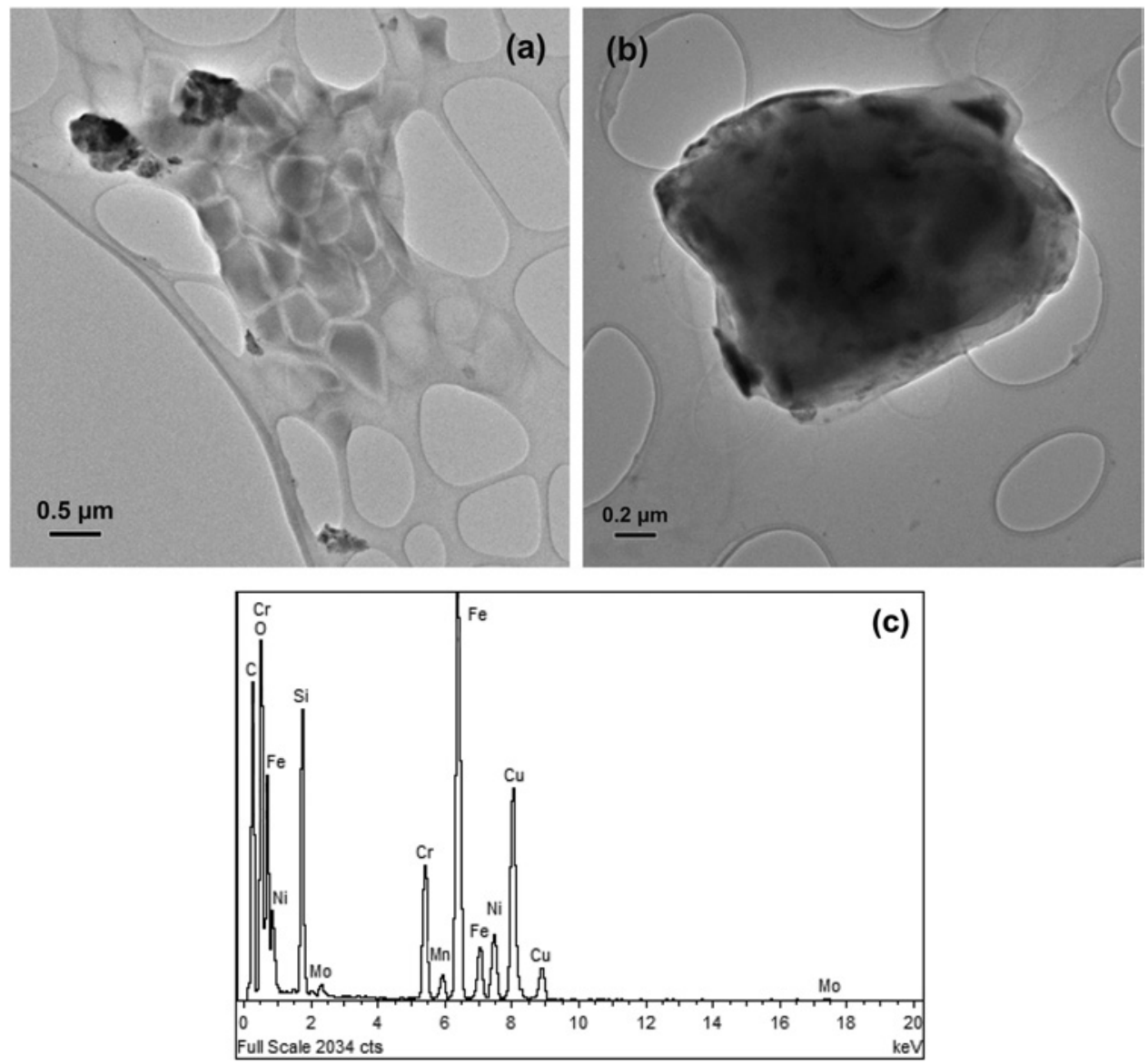

Figure 7: $(a-b)$ Small particles of silicate and steel isolated from drug product by filtration directly onto TEM grid. (c) TEM/EDS spectrum from silicate ( $\mathrm{Si}, \mathrm{O}) / \mathrm{steel}(\mathrm{Fe}, \mathrm{Cr}, \mathrm{Ni}, \mathrm{Mn}, \mathrm{Mo})$ particle.

The current work addresses the difficulty of removing thin particulate and residues from a filter for transfer to a TEM grid. Liquid suspected of containing glass delamination particulate can be representatively sampled by filtration onto a holey carbon-coated TEM grid placed on a polycarbonate filter on a standard filtration apparatus. This approach allows TEM and SEM samples to be prepared simultaneously, which adds both efficiency and consistency to isolation and characterization of delamination particulate. 
Because the amount of material examined on a TEM grid is very small, representative sampling is always a concern. There might also be doubt that filtration of several drops of liquid will isolate enough particles of interest, particularly from samples that show little to no evidence of delamination by techniques such as light microscopy and SEM, both of which sample larger volumes. Current findings shown in Table 1 demonstrate positive identification of glass delamination flakes by TEM in samples where SEM analyses gave negative results. This is likely due at least in part to the ease of identifying particles in the TEM that are too small and thin to provide sufficient contrast in the SEM. The addition of TEM to the multi-technique methodology already brought to bear on identification of glass delamination, especially when coupled with the simple grid preparation method described here, increases the likelihood of positive identifications, and may possibly flag delamination while it is still in its early stages. Complicating factors such as flaking of coating layers and formation of secondary products, as well as presence of other types of small contaminant particles, may be better identified by $\mathrm{x}$-ray spectrometry and electron diffraction in the TEM than by techniques currently employed.

\section{Conclusion}

Direct filtration of liquids onto holey carbon-coated TEM grids has been shown to be a fast and simple way in which to isolate representative samples of thin delamination flakes and residues. Characterization of materials associated with glass delamination by TEM/EDS can verify composition of isolated particulate in samples too thin or small for SEM/EDS analysis. Detection of smaller and thinner flakes easily observed in the TEM may also aid in earlier detection of glass delamination. Though SEM is of value for analysis of bulk glass and some types of associated particulate, this work demonstrates the benefit of TEM for unambiguous characterization of thin particulate and residues associated with glass delamination processes and provides an easy method for preparation of TEM grids, eliminating the difficulty of direct transfer of thin and fragile particulate and residues.

\section{References}

[1] D Haines et al., Contract Pharma 15 (2013) 92.

[2] USP General Chapter $<1660\rangle$, "Evaluation of the Inner Surface Durability of Glass Containers," U.S. Pharmacopeial Convention, Rockville, MD, 2012. http://www.usp.org/sites/default/files/usp_pdf/ EN/USPNF/revisions/c1660.pdf.

[3] EF Schumacher et al., Microsc Microanal 18(S2) (2012) 244.

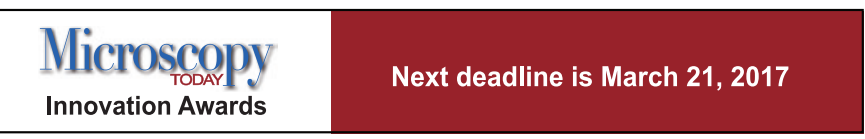

Join us for the inaugural Pre-Meeting Congress featuring the best biological and physical sciences microscopy and microanalysis research of young scientists.

The Pre-Meeting Congress highlights will include:

Friday Evening Social

Join peers for the kick-off of the Pre-Meeting Congress at Morgan Street Brewery for food, fellowship and fun!

\section{Saturday Technical Program}

Be the first to hear Award-Winning research from the M\&M 2017 Student and Postdoctoral Scholars, as well as Early Career Award Winners at America's Center Convention Complex.

\section{Networking Luncheon}

Enjoy lunch and panel discussion featuring recent graduates and industry professionals providing insight into today's job search.

\section{Saturday Reception and Dinner}

Celebrate MSA's 75th Anniversary with cocktails \& dinner in St. Louis' Ballpark Village - Great segue from the Pre-Meeting Congress to the start of M\&M 2017! 